\title{
Steady-State Harmonic Domain Matrix-Based Modeling of Four-Quadrant EMU Line Converter
}

\author{
Hui Wang ${ }^{*}$, Mingli Wu ${ }^{\dagger}$, Vassilios G. Agelidis ${ }^{* *}$, and Kejian Song* \\ ${ }^{*}$ School of Electrical Engineering, Beijing Jiaotong University, Beijing, China \\ *** Dept. of Electrical Engineering and Telecommunications, University of New South Wales, Sydney, Australia
}

\begin{abstract}
As a non-linear time variant system, the four-quadrant line converter of an electric multiple unit (EMU) was expressed by linear time periodic functions near an operating point and modeled by a steady-state harmonic domain matrix. The components were then combined according to the circuit connection and relations of the feedback control loops to form a complete converter model. The proposed modeling method allows the study of the amplitude of harmonic impedances to explore harmonic coupling. Moreover, the proposed method helps provide a better design for the converter controllers, as well as solves the problem in coordination operation between the EMUs and the AC supply. On-site data from an actual $\mathrm{CRH}_{2}$ high-speed train were used to validate the modeling principles presented in the paper.
\end{abstract}

Key words: Four quadrant line converter, Harmonic domain matrix, Harmonic impedance matrix, Harmonic transfer function, Linear time periodic system

\section{INTRODUCTION}

Modern traction systems for high-speed trains generally utilize advanced, high-voltage, high-power electronic converters. All modern high-speed trains, such as the $\mathrm{CHR}_{2}$ in China, have more than one motorized coach, which is called an electric multiple unit (EMU) [1].

The single-phase (1P) four-quadrant line converter (4Q-LC) controlled by pulse-width modulation (PWM) is capable of offering high power factor operation and energy feedback through regenerative braking. However, the AC supply currents may still contain some low- and high-order harmonics [2].

Recently, harmonic resonance events caused by system coordination issues occurred in railways, and several related technological challenges that emerged have to be understood and addressed. For example, on April 16, 2009, the extremely high harmonic resonant overvoltage caused the burnout of onboard zinc-oxide surge arrester of a $\mathrm{CRH}_{2}$ in Hefei-Wuhan high-speed line, thus causing severe delays in the train

Manuscript received Aug. 6, 2013; accepted Feb. 26, 2014

Recommended for publication by Associate Editor Se-Kyo Chung.

${ }^{\dagger}$ Corresponding Author: mlwu@bjtu.edu.cn

Tel: +8610-5168-7081, Beijing Jiaotong University

*School of Electrical Engineering, Beijing Jiaotong University, China

${ }^{* *}$ Dept. of Electrical Engineering and Telecommunications, University of New South Wales, Australia schedule. A similar event occurred in a CRH380A during the joint test of the Beijing-Shanghai high-speed line [3]. Thus, the modeling of harmonic interactions between the 4Q-LC and the AC supply should be more accurate.

The harmonics generated by EMU converter are usually analyzed with separate frequencies. However, conventional methods are inaccurate because the different frequencies are not independent but coupled by time variant elements.

A model of the harmonics generated by self-commutation static power converters and natural commutation diode rectifiers in DC electric railways was proposed in [4]. A generic treatment in the frequency domain for the $4 \mathrm{Q}-\mathrm{LC}$ was presented in [5] and [6]. A 3-D diagram for deriving specific analytical expressions of the harmonics of a three level (3L) 4Q-LC was used in [7]. However, these papers have not documented the influence of converter control parameters on harmonics generation. Although references [8] and [9] considered the relationship between converter control parameters and harmonics generation, the models in these papers do not take into account any interaction and coupling among the different harmonics. A harmonic transfer function (HTF) model of a diode converter and a single-loop control converter was reported in [10] and [11]. Despite the difference between the control strategy and the main structure of the model's system from those of $\mathrm{CRH}_{2}$, this HTF model is a promising analytical and modeling method. 


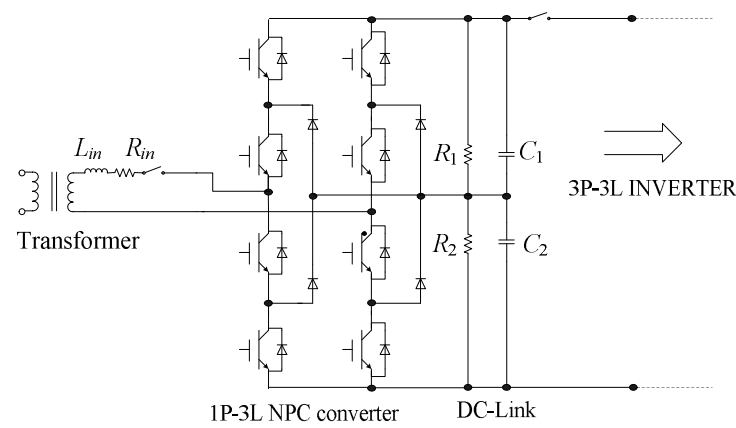

Fig. 1. Topology of 4Q-LC.

Thus far, no study has contributed to the analysis of a modern EMU 1P-3L 4Q-LC and its harmonic representation beginning from the non-linear time variant model and linearizing it around an operating point. The objective of this study is to document the harmonic domain matrix (HDM) modeling of 4Q-LC. The proposed method is capable of obtaining a numerical model of a specific 4Q-LC and analyzing its harmonic coupling. The remainder of this paper is organized as follows. In Section II, the main circuit and control strategy of the 4Q-LC is briefly introduced. In Section III, the HDM of a particular linear time periodic (LTP) system is derived and applied to the 4Q-LC. In Section IV, data measured from an actual 4Q-LC of a $\mathrm{CRH}_{2}$ train are used to confirm the validity of the modeling method. Section $\mathrm{V}$ defines harmonic impedance matrix (HIM) and explores some regular patterns that affect harmonic coupling. Finally, Section VI summarizes the conclusions of this work.

\section{Circuit AND CONTROL OF 4Q-LC}

\section{A. Main Circuit of the EMU Converter}

The $\mathrm{CRH}_{2}$ adopts power electronic converters that are designed to handle high voltage and power and fed from a secondary winding of traction transformer with a rated voltage of $1500 \mathrm{~V}$ at $50 \mathrm{~Hz}$. The control performance of the converters is crucial in achieving load dynamics, which maintains the high power factor and suppresses the harmonics.

The topology of 4Q-LC embedded in $\mathrm{CRH}_{2}$ is shown in Fig. 1 [12]. The DC link voltage is set at $3 \mathrm{kV}$. The identical capacitors $C_{1}$ and $C_{2}$ provide the neutral point to split the DC bus. The DC-link has no second order filter. Instead, the DClink capacitor values are large enough to reduce the DC voltage ripple to less than $1.5 \%$. Eliminating the DC filtering components enhances the system reliability. Two identical resistors $R_{1}$ and $R_{2}$ are utilized across each capacitor to ensure equal voltage sharing across the capacitors and to discharge the capacitors when the 4Q-LC stops functioning. To simplify the succeeding analysis, the load of 4Q-LC, which is the three phase three level $(3 \mathrm{P}-3 \mathrm{~L})$ inverter, will be modeled

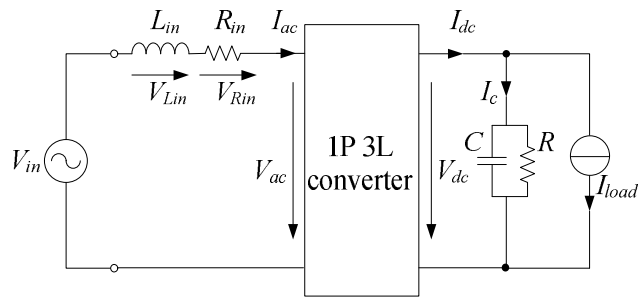

Fig. 2. The circuit of the EMU 4Q-LC.

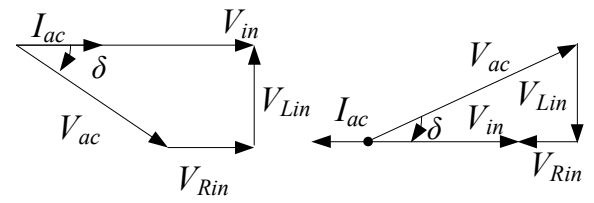

Traction mode Regenerative mode

Fig. 3. The vector diagram of a $4 \mathrm{Q}-\mathrm{LC}$.



Fig. 4. Block diagram of the instantaneous current control $\left(V_{N M}\right.$ is the amplitude of $V_{i n}$ ).

as a DC current source given that this paper focuses on $4 \mathrm{Q}-$ LC.

\section{B. Control Strategy of the Converter}

Many control strategies can be implemented for the EMU converter, including indirect current control [13], instantaneous current control [14], predictive current control [15], [16], and hysteresis current control [17]. All these strategies aim to maintain a high $\mathrm{AC}$ power factor and to make the waveform of the $\mathrm{AC}$ current be as close to a pure sinusoidal waveform as possible. These control strategies must simultaneously regulate the DC voltage under various load operating conditions.

The simplified circuit of a 4Q-LC and its vector diagram are shown in Fig. 2 and 3, respectively. In Fig. 2, the equivalent capacitor $C$ is derived from the series connection of $C_{1}$ and $C_{2}$; hence $C$ is $C_{1} / 2$. The equivalent resistor $R$ is the series combination of $R_{1}$ and $R_{2}$; hence $R$ is equal to $2 R_{1}$.

In general, $V_{i n}$ and $I_{a c}$ are in phase in the traction mode, and out of phase by $180^{\circ}$ in the regeneration breaking mode.

According to [14], instantaneous current control strategy is adopted for the 4Q-LC of CRH2, whose block diagram is given in Fig. 4. The equation of the controller is given by

$$
V_{a c}^{*}=V_{i n}-\omega L_{i n} I_{a c}^{*} \cos \omega t-R_{i n} I_{a c}^{*} \sin \omega t-K_{p i}\left(I_{a c}^{*} \sin \omega t-I_{a c}\right)
$$


Thus, $V_{a c}^{*} / V_{d c}$ can be used as the modulation waveform for comparison with the carrier. In addition, the pulse signal of the insulated-gate bipolar transistors (IGBTs) can be generated with control module for DC-link capacitor voltage balance.

\section{4Q-LC HARMONIC DOMAIN MATRIX}

The 4Q-LC in an EMU is a non-linear time variant system because of the switching devices. This system can be linearized around a steady-state periodic trajectory, and can thus be analyzed as an LTP system.

\section{A. HDM of LTP System}

Assuming the impulse response of an LTP system is $h(t, \tau)$, the output can thus be expressed as [10]:

$$
y(t)=\int_{-\infty}^{+\infty} h(t, \tau) u(\tau) d \tau
$$

Applying Laplace transform to (2) and with proper derivation (provided in the appendix), the following equation is obtained:

$$
Y(s)=\sum_{k=-\infty}^{+\infty} \hat{H}_{k}\left(s-j k \omega_{0}\right) U\left(s-j k \omega_{0}\right)
$$

where $\omega_{0}$ is the fundamental frequency, $U(s)$ is the Laplace transformation of $u(t)$, and $\hat{H}_{k}(s)$ is the Fourier coefficient of the $H(s, t)$, which is defined in the appendix as well. To simplify (3), a column vector of the input is assumed as:

$$
\begin{aligned}
\boldsymbol{U}(\mathrm{s}) & =\left[\begin{array}{llllll}
\ldots & U\left(s-j \omega_{0}\right) & U(s) & U\left(s+j \omega_{0}\right) & \ldots
\end{array}\right]^{T} \\
& =\left[\begin{array}{llllll}
\ldots & U_{-1} & U_{0} & U_{1} & \ldots
\end{array}\right]^{T}
\end{aligned}
$$

Correspondingly, the output can be expressed as:

$$
\begin{aligned}
\boldsymbol{Y}(\mathrm{s}) & =\left[\begin{array}{llllll}
\ldots & Y\left(s-j \omega_{0}\right) & Y(s) & Y\left(s+j \omega_{0}\right) & \ldots
\end{array}\right]^{T} \\
& =\left[\begin{array}{llllll}
\ldots & Y_{-1} & Y_{0} & Y_{1} & \ldots
\end{array}\right]^{T}
\end{aligned}
$$

Then, a matrix $\tilde{\boldsymbol{H}}(s)$ with elements:

$$
\tilde{\boldsymbol{H}}_{m, n}(s)=\hat{H}_{m-n}\left(s+j n \omega_{0}\right)
$$

can be obtained to determine the relation of the output to the input, which is of the Toeplitz matrix [11]. Therefore, (3) can be written as

$$
\boldsymbol{Y}(s)=\tilde{\boldsymbol{H}}(s) \boldsymbol{U}(s)
$$

$\tilde{H}(s)$ is called the harmonic transfer function (HTF) of the LTP system [7], [8].

Likewise, the input and output of an LTP system can be expressed by superposing exponentially modulated periodic (EMP) signals as in [18]:

$$
\left\{\begin{array}{l}
y(t)=e^{s t} \sum_{n} Y_{n} e^{j n \omega_{0} t} \\
u(t)=e^{s t} \sum_{m} U_{m} e^{j m \omega_{0} t}
\end{array}\right.
$$

where $s$ represents the complex modulated frequency. If the final steady-state solution of the output is required and the input signal is assumed to be a periodic signal, $s$ can be treated as 0 . Through this simplification, matrix $\boldsymbol{H}(s)$ will be transformed to a numerical matrix $\boldsymbol{H}(0)$ which represents the steady-state relation of the output to the input. Thus, in this paper, the matrix $\tilde{H}(0)$ is defined as the HDM. The HDM concept has been previously applied to the harmonic analysis of power systems in [19] and [20].

The matrix $\tilde{H}(0)$ has infinite dimensions, and therefore a LTP system can be considered as a linear time-invariant (LTI) system with infinite inputs and outputs. The Fourier coefficients of a periodic input signal are mapped by $\boldsymbol{H}(0)$ to those of the output signal. As a result, $\boldsymbol{H}(0)$ contains all the information on the coupling among different frequencies. In this paper and for practical purposes, an integer $N$ will be set as the highest harmonic order that will be taken into account to allow for the truncation of the infinite matrix into a finite one with $N \times N$ elements. Therefore, the LTP system can be viewed as a coupled multiple inputs and multiple outputs (MIMO) LTI system in the frequency domain as well. In literature, several studies have focused on the areas of signal process and control, such as [21] and [22], which analyzed LTP system through the frequency domain method.

\section{B. Calculation of HDMs for Typical Systems}

1) LTI system: The HDM of an LTI system with a transfer function $G(s)$ is a diagonal matrix that has $G\left(j n \omega_{0}\right)$ as the diagonal elements.

2) Multiplication system: A multiplication system has a system equation of

$$
y(t)=h(t) u(t)
$$

where $y(t), h(t)$, and $u(t)$ are periodic functions with the same fundamental frequency $\omega_{0}$. These three signals can then be expressed by Fourier series such as (8) with the assumption that $s$ equals to 0 . Thus, (9) is converted to

$$
\sum_{n} Y_{n} e^{j n \omega_{0}}=\sum_{n} \sum_{m} \hat{H}_{n-m} U_{m} e^{j n \omega_{0}}
$$

where $Y_{n}, \hat{H}_{n-m}$, and $U_{m}$ are Fourier coefficients of $y(t), h(t)$ and $u(t)$, respectively. Eq. (10) demonstrates that calculation can be performed without the term $e^{j n \omega_{0}}$ but only the Fourier coefficient vectors in (4) and (5) of the input and output. Hence, HDM will have the Teoplitz form:

$$
\left[\begin{array}{ccccc}
\ddots & \cdots & \cdots & \cdots & \cdots \\
\vdots & H_{0} & H_{-1} & H_{-2} & \vdots \\
\vdots & H_{1} & H_{0} & H_{-1} & \vdots \\
\vdots & H_{-2} & H_{1} & H_{0} & \vdots \\
\vdots & \ldots & \ldots & \ldots & \ddots
\end{array}\right]
$$


3) Connection of two systems: When the two above systems are connected together, the resulting HDM can then be derived. If the two systems are in series, their HDMs should be multiplied; if the systems are in parallel, their HDMs should be added. With these considerations, the HDM of a 4Q-LC can be calculated, as discussed in the succeeding section.

\section{Deriving the HDM of a $4 Q-L C$}

The components of a 4Q-LC can be classified into two types. The passive devices, including $R_{i n}, L_{i n}, R$, and $C$ shown in Fig. 2, are components of LTI systems. While the 1P-3L converter and the controller should be treated as LTP systems.

1) Passive Devices of $4 Q-L C$ : According to the discussion in Part B of Section II, the crucial work in obtaining the HDM of an LTI system is determine its transfer function $G(s)$.

- The $G(s)$ of the leakage impedance of the EMU transformer is:

$$
I_{a c}(s)=\frac{1}{R_{i n}+s L_{i n}}\left(V_{i n}-V_{a c}\right)
$$

- Given that the DC-link has no second-order filter, the $G(s)$ of the capacitor and the parallel connected discharge resistor of the DC-link is:

$$
V_{d c}(s)=\frac{R}{s R C+1} I_{c}=\frac{R}{s R C+1}\left(I_{d c}-I_{\text {load }}\right)
$$

2) $1 P-3 L$ converter of $4 Q-L C$ : For the multiplication system, obtaining the HDM is crucial in defining the periodic function $\mathrm{h}(\mathrm{t})$ in (9). The ideal description of a $1 \mathrm{P}-3 \mathrm{~L}$ converter is achieved through the modulation function $\mathrm{m}(\mathrm{t})$ defined by

$$
V_{a c}(t)=m(t) V_{d c}(t)
$$

Supposing no power is lost within the converter, the relationship between the input and output currents can be identified by applying the power balance principle:

$$
I_{d c}(t)=m(t) I_{a c}(t)
$$

The linearization of (14) and (15) around a certain steadystate operating point are given by

$$
\left\{\begin{array}{l}
\Delta V_{a c}(t)=m^{0}(t) \Delta V_{d c}(t)+V_{d c}^{0}(t) \Delta m(t) \\
\Delta I_{d c}(t)=m^{0}(t) \Delta I_{a c}(t)+I_{a c}^{0}(t) \Delta m(t)
\end{array}\right.
$$

where $m^{0}(t), V_{d c}^{0}(t)$ and $I_{a c}^{0}(t)$ are steady-state periodic signals corresponding to the given operating condition. They can be determined either by the simulation or the experimental measurement [23].

3) Controller of $4 Q-L C$ : The converter controller includes an outer voltage and an inner current control loops. Its output is the modulation function:

$$
m(t)=\frac{V_{a c}^{*}(t)}{V_{d c}(t)}
$$

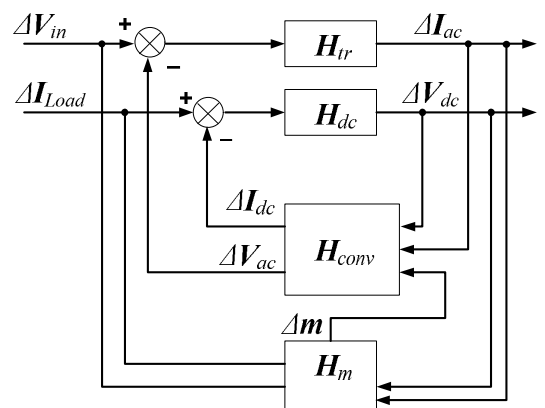

Fig. 5 The interconnection of all HDMs for a 4Q-LC.

where $V_{a c}^{*}$ is obtained from the inner current control of (1). This expression is of non-linear and should be linearized as:

$$
\Delta m(t)=-\frac{V_{a c}^{* 0}(t)}{\left(V_{d c}^{0}(t)\right)^{2}} \Delta V_{d c}(t)+\frac{1}{V_{d c}^{0}(t)} \Delta V_{a c}^{*}(t)
$$

around a steady-state operating point. To eliminate $\Delta V_{a c}^{*}(t)$, which is just an interim variable, eq. (18) should be reorganized with the specific controller equation of (1). Then $\Delta m(t)$ can be expressed only by the input and output variables $\Delta V_{\text {in }}(t), \Delta I_{\text {load }}(t), \Delta I_{a c}(t)$ and $\Delta V_{d c}(t)$ :

$$
\Delta m(t)=a \Delta V_{\text {in }}(t)+b \Delta I_{\text {load }}(t)+c \Delta I_{a c}(t)+d \Delta V_{d c}(t)_{(19)}
$$

where $a, b, c$, and $d$ are coefficients. Consequently, the HDM of this controller is the combination of the LTI and the LTP systems.

Finally, these HDMs can be connected by the manner by which they are interrelated, as shown in Fig. 5 [matrix $H_{t r}$, $H_{d c}, H_{c o n v}$, and $H_{m}$ are HDMs and obtained from (12), (13), (16), and (19) respectively]. Hence, a unified global HDM of the entire 4Q-LC can be obtained.

\section{VERIFICATION OF HDM}

Given that the actual controller parameters of the $\mathrm{CRH}_{2}$ converter are confidential because of commerciality, 4Q-LC can simply be analyzed as a grey box based on the publicly available information of its control strategy in Fig. 4 and the parameters of its main circuits, which are listed in Table I.

\section{A. Time Domain Simulation}

To validate the HDM model, the inner variables $V_{d c}$ and $I_{\text {load }}$, which are difficult to obtain in a running EMU, should be used at a given operating steady-state. In this paper, a time-domain simulation model through Matlab/Simulink should first be established. The effectiveness of this model can be verified through a comparison with the measured data.

To effectively simulate the real 4Q-LC, the controller parameters in the simulation model are designed to meet the ordinary demands of control system dynamics [24], as well as 
TABLE I

THE PARAMETERS OF THE MAIN CiRCUITS OF 4Q-LC

\begin{tabular}{|c|c|}
\hline$V_{\text {in }}($ fundamental $)=1500 \mathrm{~V}(\mathrm{rms})$ & $V_{d c}=3000 \mathrm{~V}$ \\
$L_{i n}=1.193 \mathrm{mH}$ & $C=0.008 \mathrm{~F}$ \\
$R_{\text {in }}=0.091 \Omega$ & $R=24000 \Omega$ \\
\hline
\end{tabular}

TABLE II

COMPARISON OF RESPONSE GENERATED BY TIME DOMAIN Simulation MODEL AND HDM MODEL

\begin{tabular}{|c|c|c|c|c|c|}
\hline \multicolumn{2}{|c|}{$\begin{array}{c}\text { Response } \\
\text { (harmo-nic } \\
\text { order) }\end{array}$} & \multicolumn{2}{|c|}{$\begin{array}{c}\text { simulation model } \\
\text { (harmonic phasor) }\end{array}$} & \multicolumn{2}{c|}{$\begin{array}{c}\text { HDM model } \\
\text { (harmonic phasor) }\end{array}$} \\
\cline { 2 - 6 } & $\begin{array}{c}\text { input voltage } \\
\text { disturbance }\end{array}$ & $\begin{array}{c}\text { load current } \\
\text { disturbance }\end{array}$ & $\begin{array}{c}\text { input voltage } \\
\text { disturbance }\end{array}$ & $\begin{array}{c}\text { load current } \\
\text { disturbance }\end{array}$ \\
\hline \multirow{2}{*}{$\Delta I_{a c}$} & 5 & $51.55 \angle 178.86^{\circ}$ & $53.79 \angle 1.82^{\circ}$ & $52.76 \angle 179.7^{\circ}$ & $54.09 \angle 2.94^{\circ}$ \\
& 5 & $1.71 \angle-7.98^{\circ}$ & $3.62 \angle-120.60^{\circ}$ & $1.45 \angle-9.21^{\circ}$ & $3.25 \angle-117.59^{\circ}$ \\
& 49 & $0.01 \angle-132.51^{\circ}$ & $0.01 \angle 63.23^{\circ}$ & $0.01 \angle-124.80^{\circ}$ & $0.01 \angle 60.96^{\circ}$ \\
& 51 & $0.01 \angle 35.54^{\circ}$ & $0.01 \angle-137.79^{\circ}$ & $0.01 \angle 31.97^{\circ}$ & $0.01 \angle-131.80^{\circ}$ \\
\hline \multirow{2}{*}{$\Delta V_{d c}$} & 0 & $0.00 \angle 0.00^{\circ}$ & $0.00 \angle 0.00^{\circ}$ & $0.00 \angle 0.00^{\circ}$ & $0.00 \angle 0.00^{\circ}$ \\
2 & $2.09 \angle-88.35^{\circ}$ & $4.26 \angle 170.30^{\circ}$ & $1.87 \angle-85.22^{\circ}$ & $4.49 \angle 167.00^{\circ}$ \\
\hline
\end{tabular}

to make the waveform and harmonic spectrum of $I_{a c}$ to be almost identical to that of the data measured on-site. Fig. 6 provides a comparison of the measured AC current waveform and its spectrum with the results of the simulation. In the simulation, the measured line voltage is adopted as the voltage source $V_{\text {in }}$ to ensure that the simulation is as similar to the real environment as possible. Evidently, the simulated AC current waveform and its harmonic contents are almost identical to the measured ones. Therefore, the performance of the real 4Q-LC embedded in $\mathrm{CRH}_{2}$ can be represented well by the built time domain simulation model.

\section{B. Comparison of HDM Model with Time Domain Simulation}

The time domain simulation model discussed above is convenient for deriving the converter inner variables and for superimposing specific input disturbances. Thus, this model is used to verify the HDM model.

The voltage disturbance $\Delta V_{i n}(t)$ with amplitude of $100 \mathrm{~V}$ ( $4.71 \%$ of $\left.V_{i n}\right)$ with the same phase of $V_{i n}(t)$ at fundamental frequency and a load current increase $\Delta I_{\text {load }}(t)$ of $20 \mathrm{~A}$ (4.61\% of the full load current $433.8 \mathrm{~A}$ ) are used to be injected into the 4Q-LC. The response of $\Delta I_{a c}(t)$ and $\Delta V_{d c}(t)$ are observed. Some of the primary harmonic components generated by these two models when the responses attain the steady-state are listed in Table II. Therefore, the HDM model is proved to be valid for describing the small signal variation response of 4Q-LC around a given steady-state operating point.

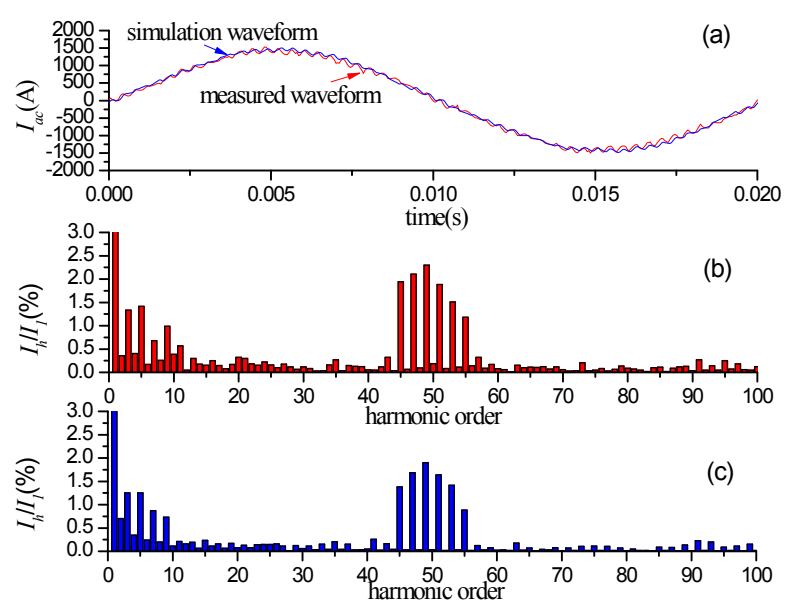

Fig. 6 Comparison of measured and simulated results. (a) The AC current waveform. (b) The measured harmonic spectrum. (c) The simulated harmonic spectrum.

\section{HARMONIC COUPLING OF 4Q-LC}

\section{A. Definition of HIM}

Although the 4Q-LC of EMU can be considered as a passive model which is described by HDM around a steadystate operating point, the definition of the coupled relation between harmonics is still fuzzy. Given that the HDM can reflect the relationship between the variations of the $\mathrm{AC}$ voltage and the current, the impedance expression that would be obtained from HDM may naturally emerge. Therefore, an HIM $\boldsymbol{Z}$ can be defined through its elements as

$$
\boldsymbol{Z}(i, j)=\frac{\Delta V_{i n}(j)}{\Delta I_{a c}(i)}
$$

where $\Delta V_{\text {in }}(j)$ represents the $j$ order harmonic phasor of $\mathrm{AC}$ voltage disturbance, and $\Delta I_{a c}(i)$ represents the $i$ order harmonic phasor of AC current variation, both around a particular steady-state operating point. The diagonal elements of $\boldsymbol{Z}$ comprise self-impedance, and other elements comprise mutual-impedance.

\section{B. Factors Affecting HIM}

Given that HIM reflects the harmonic coupling better, the factors that may influence the harmonic behavior can be discussed through several comparisons.

1) Factor 1-EMU operating state: In Section III, the HDM is derived around a periodic trajectory that corresponds to a specific EMU operating state. Therefore, this state is definitely related to HDM, as well as to HIM. The influence of the different operating states of $4 \mathrm{Q}-\mathrm{LC}$ on $\mathbf{Z}$ is shown in Table III.

According to the frequency range in focus, the truncated harmonic order $N$ is taken as 100 . Given that the smaller 
TABLE III

SELECTED ELEMENTS In $Z$ With DifFERENT EMU OPERATING STATE

\begin{tabular}{|l|c|c|c|}
\hline \multirow{2}{*}{$\begin{array}{c}\text { Impedance } \\
(\Omega)\end{array}$} & \multicolumn{3}{|c|}{ EMU operating state } \\
\cline { 2 - 4 } & Half traction & Full traction & $\begin{array}{c}\text { Full Regenerative } \\
\text { brake }\end{array}$ \\
\hline $\boldsymbol{Z}(1,1)$ & $3.797 \angle-179.8^{\circ}$ & $1.895 \angle-179.7^{\circ}$ & $1.918 \angle-0.0418^{\circ}$ \\
$\boldsymbol{Z}(3,3)$ & $46.92 \angle-80.02^{\circ}$ & $22.99 \angle-79.57^{\circ}$ & $25.91 \angle 104.7^{\circ}$ \\
$\boldsymbol{Z}(5,5)$ & $93.28 \angle-67.55^{\circ}$ & $44.57 \angle-66.69^{\circ}$ & $48.78 \angle 113.4^{\circ}$ \\
$\boldsymbol{Z}(3,1)$ & $300.2 \angle 14.67^{\circ}$ & $68.26 \angle 9.209^{\circ}$ & $190.2 \angle-8.342^{\circ}$ \\
$\boldsymbol{Z}(5,1)$ & $1455 \angle-153.3^{\circ}$ & $533.5 \angle 178.6^{\circ}$ & $673.8 \angle 30.45^{\circ}$ \\
$\boldsymbol{Z}(49,1)$ & $19610 \angle 138.3^{\circ}$ & $9731 \angle 124.8^{\circ}$ & $9881 \angle-38.88^{\circ}$ \\
$\boldsymbol{Z}(51,1)$ & $18499 \angle-49.42^{\circ}$ & $9427 \angle-31.97^{\circ}$ & $8057 \angle 117.7^{\circ}$ \\
\hline
\end{tabular}

impedance amplitude represents a larger interaction effect among the harmonics, the primary elements whose amplitudes are relatively smaller, as listed in Table III. The low harmonics orders, such as 3 and 5, have smaller self- and mutual impedances. Meanwhile, the high harmonic orders with large input current amplitude of 1P-3L NPC converter with carrier ratio 25 are 49 and 51 [7]. Moreover, the mutualimpedances of order 49 and 51 to the fundamental frequency are significantly smaller compared with other high order harmonics.

Several regular patterns shown in Table III can be further explored: the self-impedance angles are almost the same when EMU is in traction mode, regardless of the power level; the self-impedance angle when EMU is in the traction mode is almost opposite to the angle when EMU is in regenerative mode; and the amplitude of the self-impedance is inversely proportional to the traction power or regenerative power.

2) Factor 2: The controller parameters of $4 Q-L C$ : The steady-state AC current harmonics generated by the 4Q-LC in a specific EMU operating state is primarily determined through the PWM modulation strategy. However, the AC current harmonics variation after an $\mathrm{AC}$ voltage disturbance is closely related to some controller parameters of 4Q-LC. Notwithstanding the mutual-impedance has indistinct laws along with the change of the EMU operating state, the whole HIM demonstrates a strong and clear variation law along with the change of several controller parameters. Considering that the amplitude of $\mathbf{Z}(1,1), \mathbf{Z}(3,3)$, and $\mathbf{Z}(1,3)$ are within the close numerical range, only these three elements are included in Fig.7. Such treatment is appropriate, because $\mathbf{Z}(1,1), \mathbf{Z}(3,3)$ and $\mathbf{Z}(3,1)$ represent fundamental self-impedance, harmonic self-impedance, and mutual-impedance, respectively. Meanwhile, the other elements of $\mathbf{Z}$ can conform to similar variation trends.

As shown in Fig. 7, the amplitudes of $\boldsymbol{Z}$ decreased rapidly along with the increase in $K_{p v}$ (the proportional gain of the DC voltage PI controller in Fig. 4) or $K_{p i}$ (the proportional gain of AC current $\mathrm{P}$ controller in Fig. 4), except for fundamental self-impedance. Alternately, the phases of $\boldsymbol{Z}$



(a)

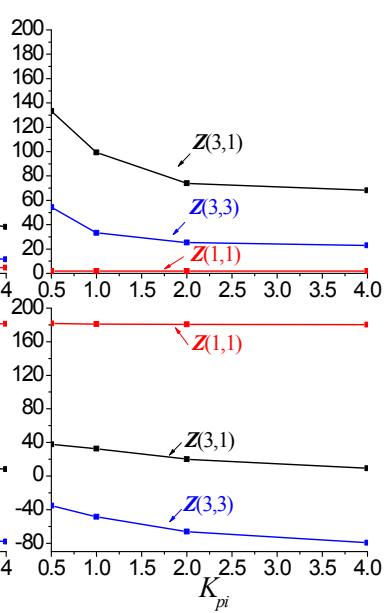

(b)
Fig. 7 The variation of typical self- and mutual-impedances at full traction state: (a) Influence of $K_{p v}$ (b) influence of $K_{p i}$.

slightly decreased as either $K_{p v}$ or $K_{p i}$ increase, except $\boldsymbol{Z}(1,1)$. Therefore, the configuration of these controller parameters can evidently affect the harmonic performance significantly.

\section{The Significance of Studying HIM}

The self-impedance of a particular 4Q-LC with any control strategy can be easily shown through the diagonal elements of the HIM, which reflects the harmonic current variation under the voltage disturbance. Although the on-site test or the time domain simulation can show the harmonic current variation under the voltage disturbance, the method proposed in the present study can reveal the effects of the operating state and control parameters on the harmonic performance conveniently with more convincing evidence compared with previous methods.

Furthermore, the harmonic coupling is clearly demonstrated by the mutual-impedance, which appears in the non-diagonal elements of HIM. For example, an AC voltage disturbance of $100 \mathrm{~V}$ at $Z(3,1)$, with the same phase of $V_{i n}(t)$ in fundamental frequency may result in about $1.5 \mathrm{~A}$ threeorder harmonic current variation at full traction state.

In designing a single 4Q-LC, its controller parameters are always maintained by dynamic performance requirements and stability analysis [24]. Moreover, with regard to HIM, the harmonic performance is an important factor that should be considered in designing controllers. Furthermore, if the harmonics cannot be controlled under the standard proportions in ordinary design process, the harmonic current offset loops should be added into the controller. However, achieving a detailed controller design is beyond the scope of the current study.

When EMUs and the power supply network are analyzed as a single integrated system, with the consideration that the HIM can reflect harmonic current variation generated by the input voltage disturbance of $4 \mathrm{Q}-\mathrm{LC}$, the harmonic 
interactions between an EMU and the power supply system, as well as the interaction among the different EMUs in the same supply section, can be investigated in a more detailed and rigorous manner. Thus, the coordinate operating issues, including harmonic resonance and the influence of the background harmonics, can be predicted by combining the HIMs of involved 4Q-LCs and the HIM of the power supply network.

\section{CONCLUSIONS}

An EMU 4Q-LC is analyzed as a specific LTP system around a periodic trajectory. This paper describes the HDM method of modeling a 4Q-LC. The HIM is likewise defined through some calculations of the HDM. The 4Q-LC can be treated as a passive impedance matrix when analyzing harmonic current variations under the line voltage disturbance around a steady-state operating point.

Through the comparison, some HIM rules are explored, which means that the regular patterns of how harmonics interact with one another can be determined. Moreover, the HIM can be treated as an additional consideration of the controller design, which can help reduce the harmonic coupling. The HDM modeling method can be easily applied to different types of electric trains; therefore, the proposed method has considerable potential to solve the harmonic coordination operation problems between electric trains and the power supply system.

\section{APPENDIX}

The output of an LTP system can be expressed through its impulse response $h(t, \tau)$ that is a $T$-periodic function with $T$ being the period [10]. Thus,

$$
h(t, \tau)=h(t+T, \tau+T)
$$

$u(\tau)$ in (2) can be expressed through the inverse Laplace transform:

$$
u(\tau)=\frac{1}{2 \pi j} \int_{\delta-j \infty}^{\delta+j \infty} e^{s \tau} U(s) d s
$$

where $U(s)$ is the Laplace transform of $u(\tau)$. Then, (2) can be rewritten by reorganizing the integral order as

$$
\begin{aligned}
y(t) & =\int_{-\infty}^{+\infty} h(t, \tau) \frac{1}{2 \pi j} \int_{\delta-j \infty}^{\delta+j \infty} e^{s \tau} U(s) d s d \tau \\
& =\frac{1}{2 \pi j} \int_{\delta-j \infty}^{\delta+j \infty} U(s) \int_{-\infty}^{+\infty} h(t, \tau) e^{s \tau} d \tau d s
\end{aligned}
$$

Assuming a periodic function $H(s, t)$ :

$$
H(s, t)=e^{-s t} \int_{-\infty}^{+\infty} h(t, \tau) e^{s \tau} d \tau
$$

In addition, the periodic function can be expressed by Fourier series with fundamental frequency $\omega_{0}$ :

$$
H(s, t)=\sum_{k} \hat{H}_{k}(s) e^{j k \omega_{0} t}
$$

where $\hat{H}_{k}(s)$ is a Fourier coefficient and can be obtained by

$$
\hat{H}_{k}(s)=\frac{1}{T} \int_{0}^{T} H(s, t) e^{-j \omega_{0} t} d t
$$

Therefore, (23) can be rewritten as

$$
\begin{aligned}
y(t) & =\frac{1}{2 \pi j} \int_{\delta-j \infty \infty}^{\delta+j \infty} \sum_{k} \hat{H}_{k}(s) e^{\left(s+j k \omega_{0}\right) t} U(s) d s \\
& =\frac{1}{2 \pi j} \int_{\delta-j \infty}^{\delta+j \infty} e^{s t} \sum_{k} \hat{H}_{k}\left(s-j k \omega_{0} t\right) U\left(s-j k \omega_{0} t\right) d s
\end{aligned}
$$

Eq. (27) is just a standard inverse Laplace transform of $Y(s)$, thus Eq. (3) can be obtained.

\section{ACKNOWLEDGMENT}

This work was supported by the Chinese Central Universities Fundamental Research Funds under Grant 2010JBZ009, 2012JBZ006, and 2011YJS069.

\section{REFERENCES}

[1] S. M. Liu, D. X. Chen, Q. L. Li, X. D. Zhang, and Y. X. Peng, "The impact of $350 \mathrm{~km} / \mathrm{h}$ high-speed railway to grid power quality," in Proc. Asia-Pacific Power and Energy Eng. Conf., pp. 27-29, 2012.

[2] S. B. Yang, M. Huang, and M. L. Wu, "High speed EMU modeling based on the measured data," in Proc. Intern. Conf. on Sustainable Power Generation and Supply, pp. 1,4,6-7, 2009.

[3] J. Q. Liu, Q. L. Yang, and Q. L. Zheng, "Harmonic analysis of traction networks based on the CRH380 series EMUs accident," in Proc. Intern. Transportation Electrification Conf. and Expo, pp. 18-20, 2012.

[4] P. Ferrari, M. Giuliari, A. Pozzobon, and P. Pozzobon, "A method for integrated harmonic analysis of multi-converter systems," in Proc. 8th Intern. Conf. On Harmonics and Quality of Power, pp. 426-431, 1998.

[5] J. Mayordomo, M. Lopez, R. Asensi, L. Beites, J. Bueno, and J. Rodriguez, "A general treatment of traction PWM converters for load flow and harmonic penetration studies," in Proc. 8th Intern. Conf. on Harmonics and Quality of Power, pp. 685-692, 1998.

[6] J. Taufiq and J. Shen, "Frequency domain modelling of traction PWM converters," in Proc. Fifth European Conf. on Power Electronics and Applications, pp. 63-67, 1993.

[7] G. W. Chang, H.-W. Lin, and S.-K. Chen, "Modeling characteristics of harmonic currents generated by highspeed railway traction drive converters," IEEE Trans. Power Del., Vol. 19, No. 2, pp. 766-773, 2004.

[8] B. Smith, N. Watson, A. Wood, and J. Arrillaga, "Steadystate model of the AC/DC convertor in the harmonic domain," IEE Proc. Generation, Transmission and Distribution, Vol. 142, No. 2, pp. 109-118, 1995. 
[9] B. Puskaric, "Modeling of single phase four quadrant converter using a multifrequency averaging approach," in Proc. 35th Annual Conf. of IEEE Industrial Electronics, IECON'09, pp. 639-644, 2009.

[10] E. Mollerstedt and B. Bernhardsson, "A harmonic transfer function model for a diode converter train," in Power Engineering Society Winter Meeting, IEEE, pp. 957-962, 2000.

[11] E. Mollerstedt and B. Bernhardsson, "Out of control because of harmonics-an analysis of the harmonic response of an inverter locomotive," IEEE Control Systems, Vol. 20, No. 4, pp. 70-81, 2000.

[12] C. Dietrich, S. Gediga, M. Hiller, R. Sommer, and H. Tischmacher, "A new $7.2 \mathrm{kV}$ medium voltage 3-level-NPC inverter using 6.5 kV-IGBTs," in European Conf. Power Electronics and Applications, pp. 1-9, 2007.

[13] J.-H. Choi, H.-C. Kim, and J.-S. Kwak, "Indirect current control scheme in PWM voltage-sourced converter," in Proc. Power Conversion Conference-Nagaoka, pp. 277282, 1997.

[14] Y. Nishida, O. Miyashita, T. Haneyoshi, H. Tomita, and A. Maeda, "A predictive instantaneous-current PWM controlled rectifier with AC-side harmonic current reduction," IEEE Trans. Ind. Electron., Vol. 44, No. 3, pp. 337-343, Jun. 1997.

[15] P. Cortes, M. P. Kazmierkowski, R. M. Kennel, D. E. Quevedo, and J. Rodríguez, "Predictive control in power electronics and drives," IEEE Trans. Ind. Electron., Vol. 55, No. 12, pp. 4312-4324, Dec. 2008.

[16] M. Perez, J. Rodriguez, and A. Coccia, "Predictive current control in a single phase PFC boost rectifier," in IEEE Intern. Conf. Industrial Technology, pp. 1-6, 2009.

[17] C. Gatlan and L. Gatlan, "AC to DC PWM voltage source converter under hysteresis current control," in Proc. the IEEE Intern. Symposium on Industrial Electronics, pp. 469-473, 1997.

[18] N. M. Wereley and S. R. Hall, "Frequency response of linear time periodic systems," in Proc. 29th IEEE Conf. on Decision and Control, pp. 3650-3655, 1990.

[19] J. Arrillaga, A. Medina, M. Lisboa, M. Cavia, and P. Sanchez, "The harmonic domain. A frame of reference for power system harmonic analysis," IEEE Trans. Power Syst., Vol. 10, No. 1, pp. 433-440, Feb. 1995.

[20] E. Acha, J. Arrillaga, A. Medina, and A. Semlyen, "General frame of reference for analysis of harmonic distortion in systems with multiple transformer nonlinearities," in IEE Proc. C (Generation, Transmission and Distribution), pp. 271-278, 1989.

[21] N. Bayan and S. Erfani, "Frequency analysis of linear time-varying systems: A new perspective," in Circuits and Systems, 2005. 48th Midwest Symposium on, pp. 14941497, 2005.

[22] H. Kano and T. Nishimura, "Controllability, stabilizability, and matrix Riccati equations for periodic systems," IEEE Trans. Autom. Control, Vol. 30, No. 11, pp. 1129-1131, Nov. 1985.
[23] A. Semlyen, E. Acha, and J. Arrillaga, "Harmonic Norton equivalent for the magnetising branch of a transformer," in IEE Proc. C Generation, Transmission and Distribution, pp. 162-169, 1987.

[24] P. A. Dahono, Y. R. Bahar, Y. Sato, and T. Kataoka, "Damping of transient oscillations on the output LC filter of PWM inverters by using a virtual resistor," in 4th IEEE Intern. Conf. Power Electronics and Drive Systems, pp. 403-407, 2001.



Hui Wang was born in Tianjin, China, in 1985. He received his B.Sc. degree in Electrical Engineering from Huazhong University of Science and Technology. He is currently working on his Ph.D. in Electrical Engineering at Beijing Jiaotong University. His research interests include the mechanism and conditions of low frequency oscillations of traction power systems and the control strategy of four-quadrant EMU converters.

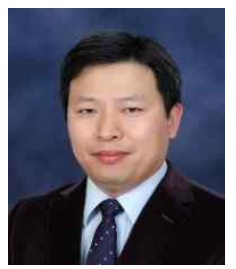

Mingli Wu was born in Hebei Province, China, on November 11, 1971. He received his B.Sc. and M.Sc. degrees in Electrical Engineering from Southwest Jiaotong University, Chengdu, China, in 1993 and 1996, respectively, and his Ph.D. in Electrical Engineering form Beijing Jiaotong University, Beijing, China, in 2006. Since 2008, he has been a Professor at the School of Electrical Engineering, Beijing Jiaotong University. His research areas include power supply for electric railways, digital simulation of power systems, and electric power quality.

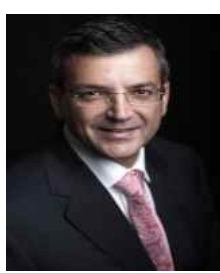

Vassilios G. Agelidis (SM'00) was born in Serres, Greece. He received his B.Eng. degree in Electrical Engineering from Democritus University of Thrace, Greece, his M.S. degree in Applied Science from Concordia University, Canada, and his Ph.D. in Electrical Engineering from the Curtin University of Technology, Australia. He is currently the Director of the Australian Energy Research Institute (AERI) at the University of New South Wales. He is a visiting research professor at the School of Electrical Engineering, Beijing Jiaotong University. From 2003 to 2005, he served as an Associate Editor of the IEEE POWER ELECTRONICS LETTERS and the Chair of the PELS Chapter Development Committee. He was an AdCom member of IEEE PELS from 2007 to 2009 and the Technical Chair of the $39^{\text {th }}$ IEEE PESC'08, in Rhodes, Greece.

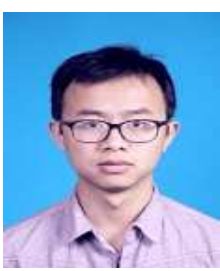

Kejian Song was born in Hunan Province, China, on January 8, 1988. He received his B.Sc. degree in Electrical Engineering from Shaoyang University, Shaoyang, China, in 2010 and his M.Sc. degree in Electrical Engineering from Beijing Jiaotong University, Beijing, China, in 2012. Since 2012, he has been pursuing his Ph.D. in Electrical Engineering at Beijing Jiaotong University. His research areas include the power quality of traction power supply systems and traction converters. 